\title{
Nanoscale mechanical properties of lipid bilayers and their relevance in biomembrane organization and function
}

\author{
Andrea Alessandrini ${ }^{\mathrm{a}, \mathrm{b}}$, Paolo Facci ${ }^{\mathrm{a}, *}$ \\ a Centro S3, CNR-Istituto Nanoscienze, Via Campi 213/A, 41125 Modena, Italy \\ b Department of Physics, University of Modena and Reggio Emilia, Via Campi 213/A, 41125 Modena, Italy
}

\section{A R T I C L E I N F O}

\section{Article history:}

Received 29 December 2011

Received in revised form 19 March 2012

Accepted 20 March 2012

\section{Keywords:}

Supported lipid bilayers

Force spectroscopy

AFM

\begin{abstract}
A B S T R A C T
The mechanical properties of biological systems are emerging as fundamental in determining their functional activity. For example, cells continuously probe their environment by applying forces and, at the same time, are exposed to forces produced by the same environment. Also in biological membranes, the activity of membrane related proteins are affected by the overall mechanical properties of the hosting environment. Traditionally, the mesoscopic mechanical properties of lipid bilayers have been studied by micropipette aspiration techniques. In recent years, the possibility of probing mechanical properties of lipid bilayers at the nanoscale has been promoted by the force spectroscopy potentiality of Atomic Force Microscopes (AFM). By acquiring force-curves on supported lipid bilayers (SLBs) it is possible to probe the mechanical properties on a scale relevant to the interaction between membrane proteins and lipid bilayers and to monitor changes of these properties as a result of a changing environment. Here, we review a series of force spectroscopy experiments performed on SLBs with an emphasis on the functional consequences the measured mechanical properties can have on membrane proteins. We also discuss the force spectroscopy experiments on SLBs in the context of theories developed for dynamic force spectroscopy experiments with the aim to extract the kinetic and energetic description of the process of membrane rupture.
\end{abstract}

(C) 2012 Elsevier Ltd. All rights reserved.

\section{Introduction}

Since the monumental work "On growth and forms" by D'Arcy Thompson (1917) the feeling that mechanical forces can determine the evolution and behavior of living systems informed more and more the approaches of physicists to the study of bio-systems. These approaches involved the mechanical characterization of tissues and the possible implications of mechanical anomalies on pathological situations (Pelling and Horton, 2008). With an increasing shift of the attention to cellular and molecular components, methods have been developed to study the mechanical properties of biological structures with an increasing spatial and force resolution (Hoffman and Crocker, 2009). Among these techniques, the introduction of Atomic Force Microscopy with its intrinsic Force Spectroscopy capabilities prompted a series of investigations in which mechanical properties of single cells, single biomolecules and supported lipid bilayers (SLBs) could be studied (Kasas and Dietler, 2008; Dufrêne et al., 1998; Garcia-Manyes and Sanz, 2010; Alessandrini and Facci, 2005). In the case of SLBs, the mechanical characterization can be extended from the micron-scale, the typical

\footnotetext{
* Corresponding author at: Centro S3, CNR-Istituto Nanoscienze, Via Campi 213/A, 41125 Modena, Italy.

E-mail address: paolo.facci@unimore.it (P. Facci).
}

range of investigation of techniques such as micropipette aspiration (Rawicz et al., 2000), to nano-scale, which is particularly relevant for its possible influence on transmembrane protein function. The possibility of facing the study of the mechanical properties of lipid bilayers by exploiting the theory of elasticity developed in the context of liquid crystals was pioneered by Helfrich (Helfrich, 1973). At the micron-scale, the calculations derived from the Helfrich theory have been verified by studying the behavior of membranes composing Giant Unilamellar Vesicles (GUVs) with the micropipette aspiration technique (Henriksen and Ipsen, 2004). The possibility that the mechanical properties of biological membranes can exert a strong influence on the behavior of integral membrane proteins has been proposed since the first evidences of the properties of mechanosensitive channels (Martinac et al., 1987; Phillips et al., 2009). This scenario points to a role of the physical properties of the bilayer, along with its chemical composition at molecular scale, in affecting membrane functions (Marsh, 2008; McIntosh and Simon, 2006; Lee, 2004; Alessandrini and Facci, 2011a). The validity of this concept is nowadays corroborated by many experimental evidences and, as such, it enables a mathematical and quantitative approach to the understanding of the behavior of membrane proteins as affected by the lipid bilayer properties. Even if it is not to be excluded that long range mechanical properties of lipid bilayers, such as those related to undulations, might have an effect on protein activity, it is of extreme relevance to measure membrane 
mechanical properties at the nanoscale. Indeed, that is the typical scale of membrane heterogeneity believed to be relevant to conformational transitions involved in the functional activities of membrane proteins (Bagatolli et al., 2010).

In recent times, the consideration that the lateral spatial heterogeneity of biological membranes might have a strong functional role re-emerged from the Seventies, when this concept was mainly used for membrane model systems (Morrisett et al., 1975; Simons and Ikonen, 1997). This concept is nowadays related to the lipid-raft hypothesis which foresees the presence in the biological membrane of nanometric and transient heterogeneities characterized by a different thermodynamic phase of lipids, usually referred to as "liquid ordered phase" (Lingwood and Simons, 2010). The concept related to phase heterogeneities is strictly connected to the process of phase transition and, in the present context, to the variations of the membrane mechanical properties among the different phases. Of major interest is also the behavior of lipid bilayers in phase transition regions due to the observation that many biological systems reside in the proximity of a phase transition or a critical point (Mora and Bialek, 2011). From a mechanical point of view, the lipid bilayer does not have exclusively elastic properties, but it is endowed with lateral viscous properties which make it similar to a two-dimensional viscous fluid in which the dissipation is mainly due to internal modes rather than to coupling with the bulk solvent. Every deformation of the lipid bilayer which might be induced by a conformational variation of integral membrane proteins will involve a flow of lipids due to a substantial volume conservation of lipid ensembles. As such, also the viscous properties of lipid bilayers have an effect on the activity of membrane proteins. This view is strongly supported by the general concepts that solvent fluctuations dominate protein activity (Beece et al., 1980; Frauenfelder et al., 2009). In the context of integral membrane proteins, the solvent is represented by the lipid bilayer whose viscosity will affect the rate of protein-related processes (Alessandrini and Facci, 2011b). The rate of these processes will be strongly connected to the viscosity of the solvent as suggested and experimentally verified by the Kramer's transition state theory for enzyme activity (Frauenfelder, 2010). The rate has a biphasic behavior as a function of the solvent viscosity with a maximum value obtained for a critical value of the viscosity. Accordingly, the membrane viscosity will represent another physical parameter capable of modulating the activity of membrane proteins besides temperature, transmembrane potential, or $\mathrm{pH}$.

AFM offers great potentialities in the study of elastic and viscous properties of lipid bilayers assembled to form membranes supported by a rigid substrate. In this context, the microscopic imaging capabilities of this technique can be coupled to its force spectroscopy potentialities. In case of supported lipid bilayers, force spectroscopy technique typically involves the study of the jumpthrough event which occurs to an AFM tip once the force applied to a lipid bilayer reaches a certain threshold (Dufrêne et al., 1998). This event, which is inherently stochastic, can reveal important properties of the lipid bilayer as a consequence of the involved molecular mechanistic event. The force threshold is affected by the temperature of the system (Garcia-Manyes et al., 2005a) or the ionic strength of the solution (Garcia-Manyes et al., 2005b), but it is also the result of a dynamic process and, as such, depends on the speed of the probing tip (Butt and Franz, 2002; Loi et al., 2002). Moreover, the dependence of the threshold-force on the tip speed allows retrieve information on the energy landscape underlying the rupture of the bilayer by the AFM tip. The relevance of this process in the biological context is connected to its analogy with pore formation events in pure lipid membranes. Measurements of the threshold-force and of the overall features of the force curve as a function of the tip-speed allows also acquiring information on the viscous properties of the lipid bilayer, establishing a connection to the aforementioned medium viscous properties in controlling the rate of reactions which involve membrane proteins.

The present review provides an overview of the potentiality of AFM for the characterization of the mechanical properties of supported lipid bilayers especially in relation to the thermodynamic state of the membrane. The relevance of these mechanical properties for the functional activities of integral membrane proteins will be highlighted and, when possible, some specific experimental evidences will be presented. The review is organized as follows. In Section 2 the specificity of the lipid bilayer model system exploited for the AFM mechanical characterization - supported lipid bilayers (SLBs) - will be presented in relation to other unsupported model systems. In Section 3 the technique of Force Spectroscopy in the context of lipid bilayers will be illustrated. In Section 4 an overview of the results from our group and from other groups will be presented together with evidences of how bilayer mechanical properties can affect protein functional activity. In Section 5 the technique of dynamic force spectroscopy employed to explore the energy landscape of AFM tip penetration in the lipid bilayer will be introduced and the results of relevant experiments will be discussed. The review will end with a section dedicated to conclusions and perspectives of these studies in relation also to their biological relevance.

\section{Supported lipid bilayers}

\subsection{Preparation of supported lipid bilayers}

Nanomechanical investigations of the mechanical properties of lipid bilayers by Atomic Force Spectroscopy are typically performed on supported lipid bilayers. The possibility of measuring the bending modulus of lipid bilayers in cases where they are suspended over nanosized holes has also been exploited (Mey et al., 2009), but in the present review we will concentrate on homogeneously supported lipid bilayers, where the focus is mainly on indentation of the bilayer and on its mechanical stability. SLB technique consists of a lipid bilayer on a rigid and typically hydrophilic substrate such as glass, silicon oxide or mica. It was initially developed by the McConnell's group to study the interaction of cells with lipid bilayers (McConnell et al., 1986; Castellana and Cremer, 2006). SLBs can be assembled by two main different strategies, each presenting specific advantages: the Langmuir Blodgett/Schaefer approach (Dufrêne et al., 1997) and the vesicle fusion technique (Brian and McConnell, 1984). The first technique is based on two consecutive transfers to a solid substrate of lipid monolayers assembled at the liquid/air interface in a Langmuir trough. The appealing feature of this approach is connected with the possibility of forming lipid bilayers characterized by a transbilayer lipid asymmetry (Picas et al., 2010), reproducing the actual situation found in biological membranes, even if the retention of the initial composition asymmetry between the two leaflets has been questioned (Crane et al., 2005). The vesicle fusion technique allows forming supported lipid bilayers from unilamellar vesicles in solution. Upon contact with surfaces, under specific conditions, unilamellar vesicles rupture, forming a planar bilayer. The implementation of the vesicle fusion technique is substantially easier than the technique based on Langmuir trough. In both preparation strategies, the presence of a thin water layer between the bilayer leaflet nearer to the substrate (proximal leaflet) and the substrate itself enables lipid diffusion (Kim et al., 2001; Johnson et al., 1991; Koenig et al., 1996). Fig. 1 shows a scheme of a supported lipid bilayer. 


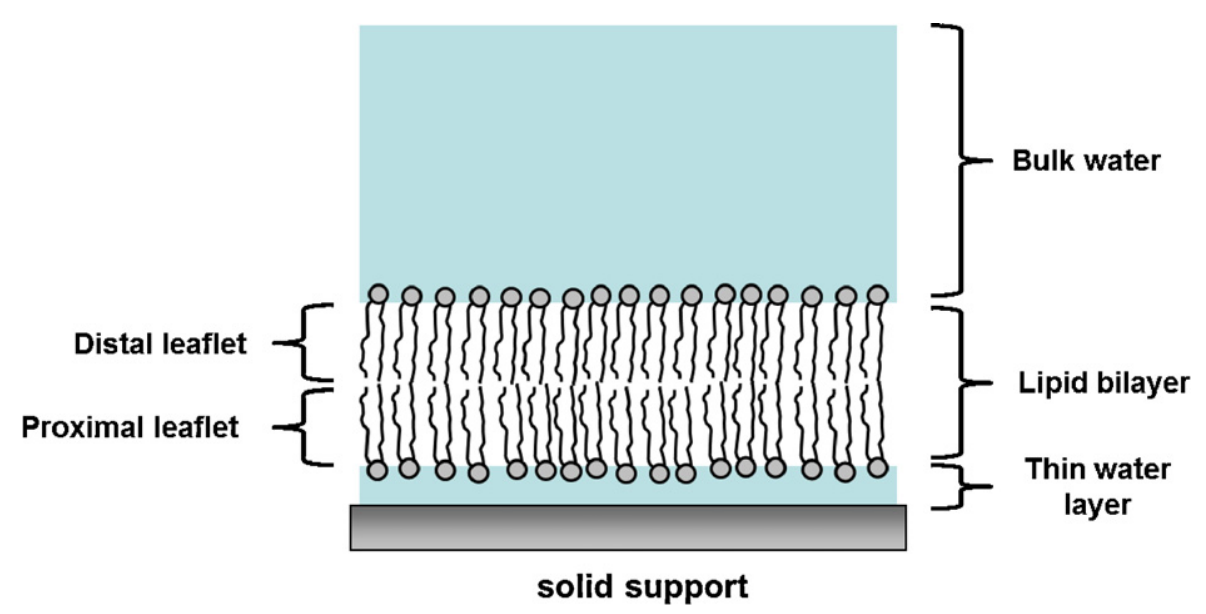

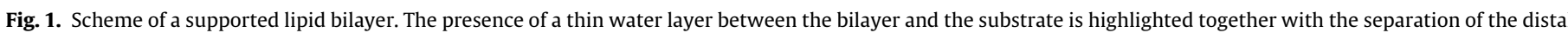
and proximal leaflets.

\subsection{Chemical-physics of supported lipid bilayers and comparison with other lipid bilayer model systems}

Due to the presence of a solid support in close vicinity of one leaflet of the lipid bilayer, the overall thermodynamic properties of SLBs are different with respect to other unsupported lipid bilayer model systems, such as unilamellar vesicles (Keller et al., 2005; Stottrup et al., 2004). The presence of the substrate might potentially have a strong influence on the asymmetry of the physical properties of the two leaflets (Deserno, 2009). In particular, recent simulation studies demonstrated that in SLBs the surface density of lipid molecules in the two leaflets differs, being higher in the leaflet nearer to the substrate (proximal leaflet) than in the one in contact with the bulk solution (distal leaflet) (Xing and Faller, 2008, 2009). The vertical density asymmetry in the bilayer can have an influence on interleaflet coupling (Collins, 2008). This coupling represents a sort of lipid signaling mechanism across the bilayer and it is, at present, the focus of many experimental and theoretical studies aimed at elucidating its principles in light of its possible biological relevance (Collins and Keller, 2008). One of the main effects of the asymmetry between the two leaflets is represented by the behavior of the bilayer at its main phase transition (Keller et al., 2005; Seeger et al., 2009a). In general, lipid bilayers display a reversible phase transition between a gel $\left(L_{\beta}\right)$ and a liquid-crystalline $\left(L_{\alpha}\right)$ phase when the temperature, or other parameters, change (e.g. pressure, $\mathrm{pH}$ ). The transition is accompanied by changes in lipid chains (from ordered to disordered) and lattice order (from solid to liquid). The change in the lipid chain order is reflected in a variation of the membrane thickness which makes the transition easily observable by AFM topographic imaging. Lipid bilayers in the form of liposomes composed by only one type of phospholipid usually display a single phase transition which is associated with a collective behavior of both leaflets. When the main phase transition of solid supported lipid bilayers is studied, even in the case of a single lipid component, two separate phase transitions might be observed (Seeger et al., 2009a,b; Feng et al., 2005; Charrier and Thibaudau, 2005; Lin et al., 2006). Their occurrence is typically associated with a separate behavior of the two leaflets. In this case, the presence of a substrate induces a decoupling of the leaflets. Dealing with the temperature at which these transitions are observed, in general one of the two transitions is observed at the same temperature as for liposomes of the same lipid composition, whereas the other transition is observed at higher temperature (Keller et al., 2005; Seeger et al., 2009a,b). In the first case the transition is attributed to the distal leaflet which shows physical properties similar to unsupported bilayers; in the second case, i.e. higher temperature phase transition, it is attributed to the proximal leaflet. The behavior described above is however affected by SLBs preparation conditions, including temperature and ionic strength of the solution (Seeger et al., 2009a,b), and by the physical and chemical nature of the support (Seeger et al., 2010a). Indeed, the assembling of SLBs at a temperature higher than that corresponding to the bilayer main phase transition, results in a transition with coupled leaflets. This behavior points to a role of the lipid density asymmetry in influencing leaflet coupling. As a result, a high difference in the density promotes decoupling, whereas a more similar density favors coupling. A possible explanation for this result refers to interdigitation between acyl chains of the lipid molecules as a major determinant for the leaflet coupling (Merkel et al., 1989). The strong dependence of the lipid bilayer behavior at the main phase transition on the preparation conditions could explain the different results in the literature. In fact, other publications found a coupled phase transition with the domains in register using similar approaches (Tokumasu et al., 2002; Yarrow et al., 2005). Another relevant aspect for the behavior of SLBs is the physical structure of the substrate. In fact, it has been shown that the substrate roughness affects the main phase transition temperature and the coupling between the two leaflets. SLBs prepared on a silicon oxide substrate shows always only one phase transition, independently of the assembling temperature, and transition temperature is very similar to that of unsupported bilayers (Seeger et al., 2010a; Kalani et al., 2007). This evidence could be rationalized on the basis of the very complicated scenario of forces involved between the substrate and the proximal leaflet such as: van der Waals forces, entropic forces due to the undulation suppression exerted by the solid surface, and hydration forces (Israelachvili, 1992).

Asymmetry between the two leaflets could also involve dynamical properties. The different lipid density and environment of the two leaflets could result in a dissimilar lateral mobility. Recent experimental results have shown that lipid diffusion in freestanding bilayers (GUV, Giant Unilamellar Vesicles) is more than twice faster than in supported lipid bilayers measured under the same conditions (Przybylo et al., 2006). An even more complicated issue is the possible difference in diffusion coefficients between the two leaflets in the case of a SLB (Hetzer et al., 1998; Zhang and Granick, 2005). In this context, the physical properties of the thin water layer between the substrate and the proximal leaflet could be very different from those of the bulk water facing the distal leaflet, thus causing asymmetry in lipid diffusion constants. Based on the above considerations it appears very difficult to compare results obtained 


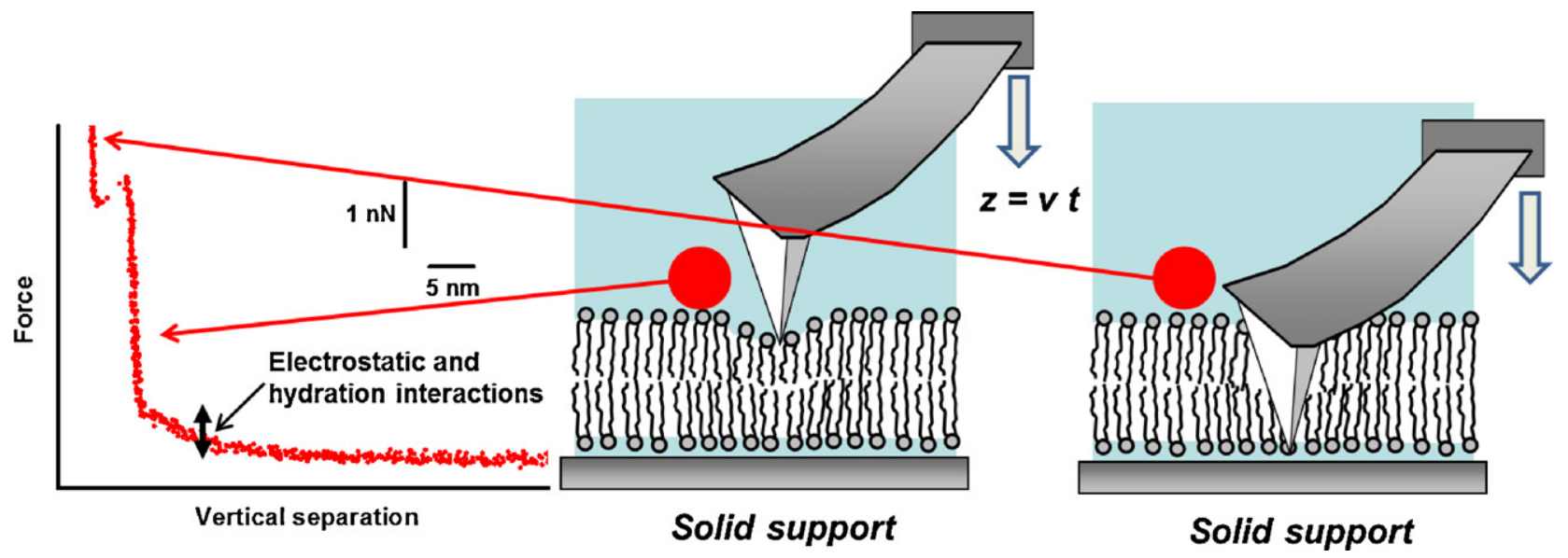

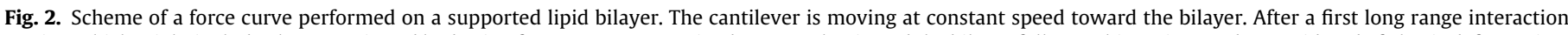

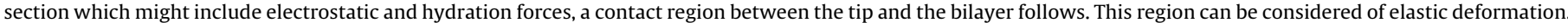
After a threshold force is reached, the tip jumps in contact with the substrate by a process of plastic deformation (rupture) of the membrane.

in different laboratories even on the same lipid system. In fact, subtle differences in sample preparation procedures could result in a different interleaflet coupling strength or surface density of the lipids, thus affecting the obtained results.

All the above aspects should be kept in mind when nanomechanical properties of SLBs are studied by AFM. Typical parameters extracted in these measurements could be affected by the state of the bilayer, namely the interleaflet coupling. Indeed, force spectroscopy experiments could also help understanding the phenomenon of interleaflet coupling by measuring the threshold force required to jump through a lipid bilayer in the case of coupled or uncoupled leaflets (Alessandrini et al., submitted for publication).

As to the relation between SLBs and unsupported lipid systems, the presence of a strong asymmetry in the nano and microenvironment of the two leaflets could in some way reproduce conditions similar to those experienced by lipids in membranes where the inner leaflet interacts strongly with the cytoskeleton. Hence, rather than being regarded as a drawback of the model, the presence of the substrate can provide further clues on the principles ruling the behavior of biological membranes such as the typical dimension of stable domains (Thormann et al., 2007).

\section{Atomic force spectroscopy on supported lipid bilayers}

A typical force curve on a supported lipid bilayer together with schemes corresponding to the different regions of the curve are reported in Fig. 2. Typically, Force Spectroscopy by AFM allows to investigate forces in the range between 10 and $10^{4} \mathrm{pN}$ (Neuman and Nagy, 2008). Other techniques such as optical tweezers or magnetic tweezers allow force ranges in the order of $10^{-2}-100 \mathrm{pN}$. AFM force spectroscopy measurements rely both on approach and retraction curves. Usually, in the case of lipid bilayers, the approach curve is used for extracting nanomechanical information but, in some cases, valuables data can also be contained in retraction curve (Dufrêne et al., 1998; Armond et al., 2011). For example, the retraction curve can provide an indication of the adhesion force between the AFM tip and the different regions of the lipid bilayer (Dufrêne et al., 1998). In particular, the surface of the AFM tip can also be modified with a monolayer exposing chemical groups to analyze specific interactions with the headgroups of the lipids. In the following we will concentrate on the interpretation of the approach curve. Starting from a large distance between the tip and the bilayer, no interaction is measured. Approaching the lipid bilayer surface, before a physical contact with the lipid bilayer being established, an interaction, electrostatic in nature, can be observed. This interaction depends on the chemical nature of the lipids constituting the bilayer and on the chemical nature of the tip surface. In the case of phospholipids constituting biological membranes the electric charge at the bilayer surface is typically negative at neutral $\mathrm{pH}$ (Cevc, 1990). The charge on the tip surface depends on tip material, but can also be modified by chemical functionalization of tip surface (Garcia-Manyes et al., 2006). Considering a clean bare silicon nitride tip, its surface is negatively charged in a large $\mathrm{pH}$ range around neutrality (Butt, 1991a). As a result of two negatively charged surfaces, the interaction force between tip and lipid bilayer turns out to be repulsive. The spatial extension of this interaction depends on ionic strength of the working solution through the Debye decay length and can reach tens of nanometers in the lowest ionic strength conditions. Just before contacting the lipid bilayer, hydration and steric forces associated with the removal of the water molecules strictly interacting with the hydrophilic headgroups can give rise to small repulsive forces (Butt, 1991b, 1992).

Once contact is established between tip and lipid bilayer, a region of elastic deformation is encountered. Many studies concentrated on the analytical description of this elastic region according to different models (Garcia-Manyes et al., 2005b; Voïtchovsky et al., 2006; Kunneke et al., 2004). In AFM indentation experiments, the usual description of the indentation-force relation is based on the Hertz model or on some of its derivations, such as the Sneddon modification (Radmacher et al., 1996; Domke and Radmacher, 1998). The Hertz model derives from an elasticity theory based on the continuum approximation of the interacting bodies (Hertz, 1882). However, several studies demonstrated the inadequacy of this model in describing the elastic deformation of lipid bilayers (Garcia-Manyes et al., 2005b; Das et al., 2010). In the case at issue molecular details, the possibility of strong spatial anisotropies, and the presence of a nearby solid support should be considered in the formulation of an analytical model of the indentation-force relation exploitable to extract quantitative parameters for the mechanical properties of the bilayer (Dimitriadis et al., 2002).

A relevant question arises, at this point, as to the relation between these mechanical parameters and those derived from micropipette experiments (Dieluweit et al., 2010). Particularly, the relevance of this question is related to the possible connections between the measured mechanical parameters and the behavior of membrane proteins. In micropipette experiments the tension applied to the membrane is in the plane of the membrane. By measuring the membrane deformation as a function of the applied tension the area stretching modulus is retrieved. This parameter associates a sort of elastic constant to the deformation of the lipid 
(a)

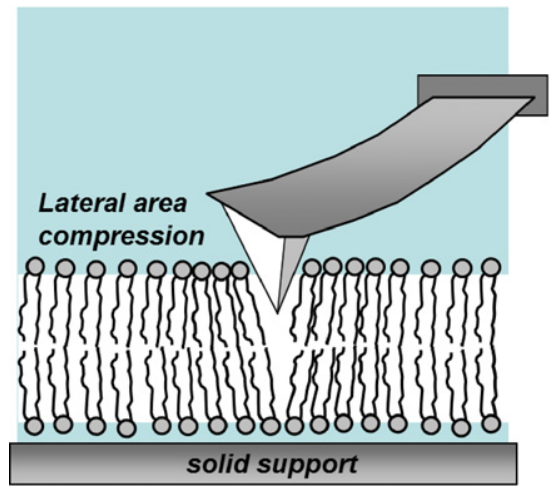

(b)

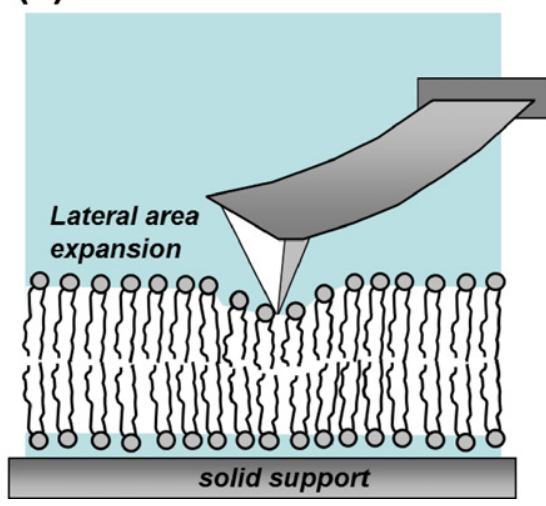

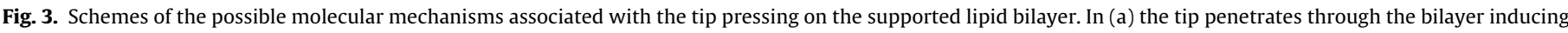

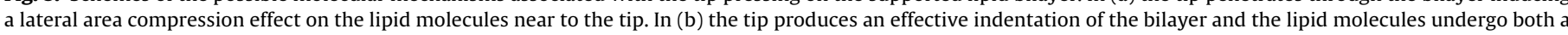
lateral area expansion and a vertical compression.

bilayer involving a variation of the area per lipid molecule. At the same time, the value of the area stretching modulus might affect the activity of all membrane proteins whose functioning mechanism involves a variation of their area in the plane of the membrane (Sachs, 2010). In a description of lipid bilayers in terms of mechanical properties also the Gibbs free-energy penalty associated with a variation of the bilayer thickness should be considered. This sort of deformation is intuitively associated with AFM indentation experiments. One should observe that, given the almost constant volume of lipid bilayers, every variation of the thickness of the bilayer involves also a variation of the area per lipid molecule. Moreover, as it will be discussed below, each of these changes involves a lateral flow of molecules which depends on bilayer viscosity and, hence, on indentation speed. The possibility of answering the previous question should encompass the understanding of the molecular lipid deformation under the force applied by the tip, probably as a function also of the tip geometry, as shown in Fig. 3. Notwithstanding this scenario is a quite complicated one, some considerations could help finding a way out. In both cases (a) and (b) of Fig. 3, tip indentation inside the lipid bilayer implies a variation of the area-per-lipid molecule. In (a), a lateral area compression is involved, whereas, in (b), an expansion. A typical approximation for the description of the mechanical parameters of the lipid bilayer in the limit of small deformations is that the energy involved in each deformation depends on the square of the deformation. This means that a harmonic spring model is assumed. As a consequence, the difference between (a) and (b) in Fig. 3 is that, even if in both cases the stretching modulus is involved, in (b) a contribution also from the compression modulus is expected. In quantitative terms, the area stretching modulus has characteristic values in the range $55-70 k_{B} \mathrm{~T} / \mathrm{nm}^{2}$ and the compression modulus has a typical value of $60 k_{B} \mathrm{~T} / \mathrm{nm}^{2}$. As a conclusion we can state that the mechanical parameter extracted from force spectroscopy experiments, either it is indicated by $E$ (Young modulus) or by $k_{A}$ (area stretching modulus), will involve the value of the area stretching modulus.

In a recent work Das et al. (2010) developed a molecular description of the bilayer indentation by an AFM tip essentially based on model (b) of Fig. 3. They derived a Gibbs free-energy cost for the lipid deformation and, taking the derivative of the energy with respect to tip indentation, the force exerted by the tip. In their derivation, they relied exclusively on the area stretching modulus and, by a fit of the derived indentation-force relation to experimental data, they obtained values for the stretching modulus similar to that obtained in micropipette experiments.

Much attention in force spectroscopy experiments on lipid bilayers has been devoted to the jump-through event which is due to a sudden jump of the tip from the surface of the deformed bilayer to the underlying substrate (Dufrêne et al., 1998; Garcia-Manyes et al., 2005a,b; Butt and Franz, 2002; Loi et al., 2002; Alessandrini et al., 2011). This phenomenon suggests a brittle behavior of the bilayer. The attention given to this aspect of the approaching curve relies on the fact that the tip penetration through the lipid bilayer resembles the formation of pores in the lipid bilayer (Butt and Franz, 2002; Garcia-Manyes et al., 2005b; Alessandrini et al., 2011). The energetics of pore formation in lipid bilayer has a great relevance in experimental situations such as electroporation. The poration process could be described in analogy to a chemical reaction: an initial state - the intact bilayer - separated from a "pored" configuration by an intermediate state of maximum Gibbs free-energy (Bier et al., 2004). Once the system is able to reach the energy required to overcome the intermediate state, the final state will be rapidly reached. Formation of pores in lipid bilayers is connected with lateral area fluctuations and the spontaneous formation of holes with a radius higher than a critical value (Glaser et al., 1988). It is to be stressed that the details of the spontaneous pore formation process differ in some aspects from the details of the pore induced by an AFM tip. In particular, pores formed in force spectroscopy experiments will be larger than the spontaneous ones, requiring higher energy to be obtained.

Also the physical and chemical properties of the substrate supporting the lipid bilayer might have a role in determining the mechanical parameters obtained by force spectroscopy experiments. As we already stated, the substrate could affect the asymmetry between the two leaflets and, in particular the surface lipid density. Apart from affecting the degree of interleaflet coupling (Seeger et al., 2010a), the surface lipid density in the leaflets could also contribute to the measurement of the mechanical parameters. In fact, the lateral area expansion modulus of the supported bilayer is strongly connected with the lipid density and the lateral pressure inside the bilayer. This particular aspect makes the comparison between experiments performed in different laboratories on the same system quite hard. In fact, it is very difficult to reproduce the same lipid surface density being this parameter affected by many environmental conditions.

In a seminal group of papers, Butt et al. studied the process of jump-through in lipid bilayers, from both a theoretical and experimental point of view (Butt and Franz, 2002; Loi et al., 2002; Franz et al., 2002). In one of the possible theoretical interpretations, the continuum nucleation model, they suggested that the force applied by the tip provides energy to favor the overcoming of the intermediate state and, by increasing the applied force, the critical radius of the fluctuations required for the hole formation decreases. Two 


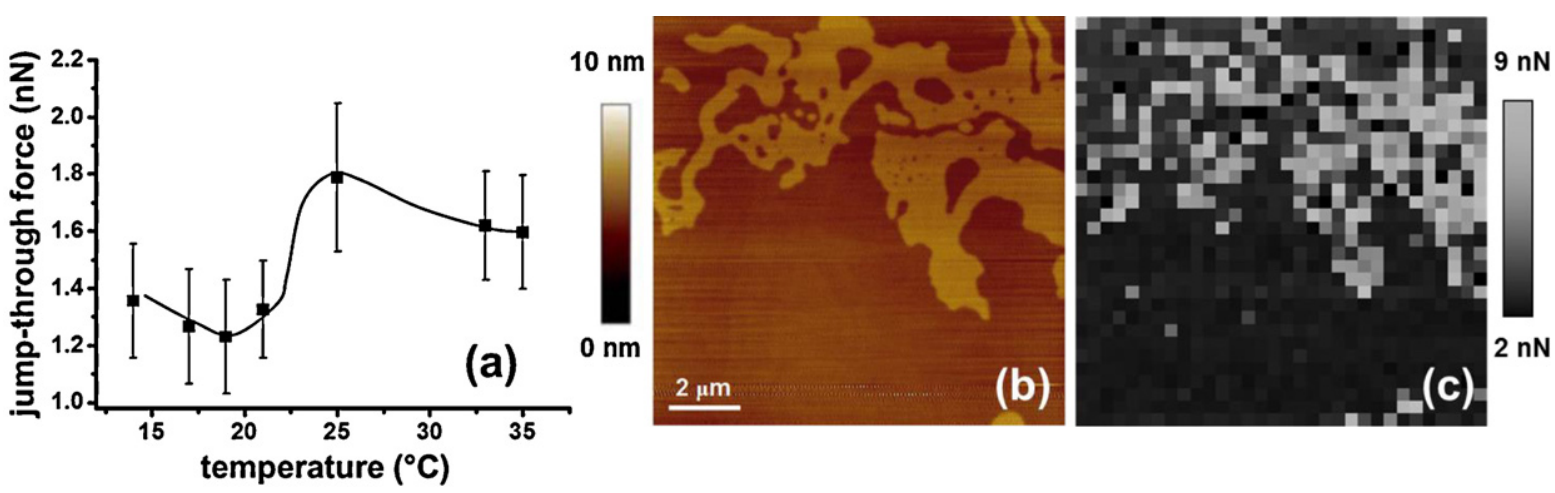

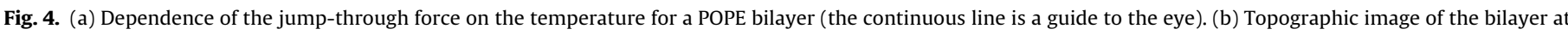

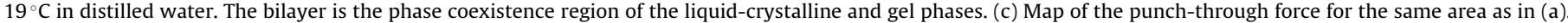
The lighter area corresponds to the gel phase of the bilayer, whereas the darker region corresponds to the still liquid-crystalline region of the bilayer.

properties of the jump-through event are relevant at this point of the discussion: the event is a kinetic process and the force value at which it occurs depends on the rate of force increase; the phenomenon is inherently stochastic and the punch-through force, even at constant rate of force increase, is distributed around a most probable value. Given the above considerations, the experimental procedure includes also aspects of dynamic force spectroscopy and it will be thoroughly discussed in a dedicated section.

\section{Overview of the force spectroscopy studies on supported lipid bilayers}

The main objective of the research involving the force spectroscopy investigation of SLBs is the determination of their mechanical properties at the nanoscale. The absolute value of the mechanical parameters is difficult to obtain due to uncertainties in the spring constant of the AFM cantilever and the choice of the right analytical model to be used in the fitting procedure. This situation is quite ubiquitous in AFM experiments on biological systems. However, as usual, changes in mechanical parameters as a consequence of variation in the thermodynamics of the system can be easily measured. At the same time, lateral variation of the parameters at nanometer scale are easily measured.

Dependence of mechanical parameters as a function of temperature (Garcia-Manyes et al., 2005a; Alessandrini et al., 2011), ionic strength of the solution and $\mathrm{pH}$ (Garcia-Manyes et al., 2005b; Alessandrini et al., 2011) has been studied. Most of the studies concentrated on the force value at which the jump-through phenomenon occurs whereas, typical elastic properties which could be extracted from the force indentation has received less attention. It is therefore more precise to define the properties related to the jump-through event as concerning "mechanical stability" rather than "mechanical properties" broadly speaking, which could suggest to think about, for example, a variation of the area compressibility modulus of the bilayer.

One of the most important parameters affecting the mechanical behavior of lipid bilayers is temperature. As we have already pointed out in Section 1, a temperature variation can induce different phase transitions in lipid bilayers according to their chemical composition. Even if temperature is a parameter which does not undergo significant changes in physiological conditions, it should be recalled that many parameters such as $\mathrm{pH}$, pressure and ionic strength are able to affect the temperature at which the phase transitions occur in lipid bilayers (Cevc, 1991). As such, the local variation of each of the above parameters is potentially able to induce localized phase transitions and it is therefore important to study how temperature variations can modify mechanical properties. Dealing with the jump-through event, if one describes it as a chemical reaction, it is to be expected the rate of the process be modified by temperature with an Arrhenius type trend. As such, an increase of the temperature will induce the transition across the intermediate state for lower force values. The description of the process by a single and constant activation energy parameter is however limited by the possible strong variation of the lipid molecules properties with temperature (Alessandrini et al., 2011). Starting from a high temperature value corresponding to a lipid bilayer in the liquid-crystalline phase, by decreasing the temperature, an increase of the jump-through force value is observed (Garcia-Manyes et al., 2005a; Alessandrini et al., 2011). However, the most significant feature of the relation between the jumpthrough force value and temperature is found when, upon a further temperature decrease, a strong decrease of the jump-through force value is observed (Fig. 4a). Exploiting the possibility of coupling microscopic and spectroscopic investigations, it has been possible to associate the decrease of the force threshold to the presence of domains still in the liquid-crystalline phase while the phase transition to the solid ordered phase was already started out, as it is shown in Fig. 4b by a topographic image of the bilayer (Alessandrini et al., 2011). Fig. 4c shows a map of the jump-through force values on the same area where the topographic map in Fig. 4b has been acquired. The image displays that the darker and thinner regions of the bilayer, corresponding to the liquid-crystalline phase, are associated with jump-through force values lower (darker) than the jump-through values on the thicker gel phase. Recalling the analogy with the formation of pores in lipid bilayers, a strong connection between the jump-through event by the AFM tip and fluctuations in the mechanical parameters of the lipid bilayer can be established. In fact, it is well established that fluctuations, for example in the area per lipid molecule, undergo a strong increase in the phase transition region (Heimburg, 1998). In the continuum nucleation model developed by Butt et al., fluctuations in the lipid positions under the tip are the starting point for the nucleation of a hole big enough to overcome the intermediate state toward the penetration of the tip through the bilayer (Butt and Franz, 2002). Accordingly, the jumpthrough force value can be considered an indication of the strength of fluctuations in the lipid bilayer (Alessandrini et al., 2011). From Fig. $4 \mathrm{c}$ it is also clear that in the liquid-crystalline domain the punch-through force has a rather homogeneous value. In the context of membrane permeability due to the formation of lipid ion-channels, two possible scenarios (not mutually incompatible) can be envisaged in the phase transition region: an increase in the probability of pore formation in the liquid domains and an increase of pore formation in the boundary regions between different phases (Heimburg, 2010). On the basis of the proportionality between the excess heat capacity and fluctuations and considering the connection between excess heat capacity and cooperativity of the phase 


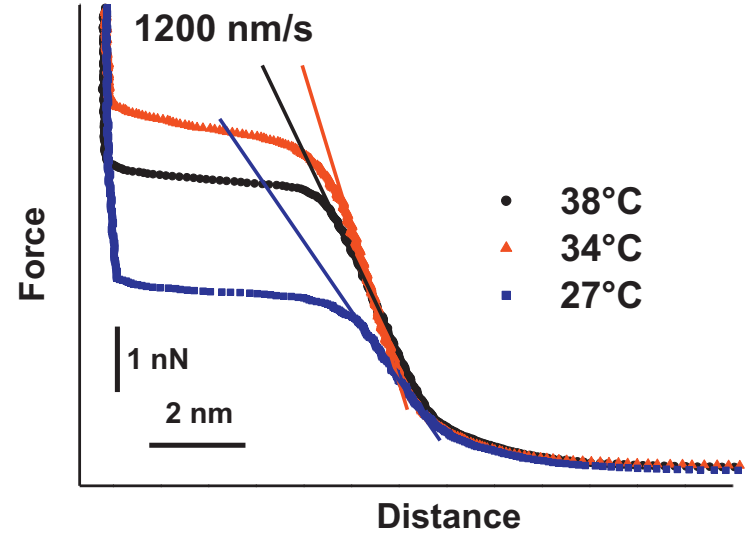

Fig. 5. Force curves obtained on a POPE supported bilayer as a function of temperature at a constant tip speed of $1200 \mathrm{~nm} / \mathrm{s}$. Each reported curve is an average of more than 200 force curves. The overlaid lines highlight the steepness of the contact region between the tip and the bilayer as an indication of the bilayer deformability. (For interpretation of the references to color in the text, the reader is referred to the web version of the article.)

transition, it appears that the formation of pores due to macroscopic fluctuations involving the whole liquid phase region is more probable (Heimburg, 2010). Here, the whole liquid-crystalline phase experiences a reduced punch-through force, without evidence of an increased pore formation probability at the domain interfaces. Performing high resolution Force Volume imaging with the aim to retrieve details of the interface region, no particular further softening at the domain boundaries is observed. A possible reason for the lack of a special behavior at the domain boundaries could lie in the interaction area of the tip with the bilayer. Probably, it is too large to allow distinguish the particular value of the punch-through force in a thin region along the phase interface.

We already pointed out in the section dedicated to the relation between force spectroscopy and mechanical parameters of the lipid bilayers, that the elastic region of interaction can provide information on the area stretching modulus of the lipid bilayer. In Fig. 5, three examples of curves obtained on a POPE bilayer at three different temperatures are reported. The black line (circles) has been measured at $38^{\circ} \mathrm{C}$ with the bilayer in the liquid-crystalline phase. A fit of the force-indentation relation proposed by Das et al. (2010) to the initial elastic region of the curve suggests a value for the area stretching modulus of $0.14 \mathrm{~N} / \mathrm{m}$. The red curve (triangles) has been obtained at $34^{\circ} \mathrm{C}$. The fit provides a value for the stretching modulus slightly higher than the value at $38^{\circ} \mathrm{C}(0.16 \mathrm{~N} / \mathrm{m})$, and the bilayer is still in the liquid-crystalline phase. The blue curve (squares) has been obtained at $27^{\circ} \mathrm{C}$ in the phase transition region of the bilayer (the curves have been acquired on the regions which are still in the liquid-crystalline phase). The area stretching modulus is in this case sensibly lower than the values obtained in the previous cases $(0.12 \mathrm{~N} / \mathrm{m})$. This finding highlights that in phase transition region not only the jump-through force is strongly reduced but also the bilayer is much more compliant.

To complete the analysis of the temperature dependence of the jump-through event, it is to be observed that the same phenomenon occurs also for the bilayer in the gel phase. The force value measured on the gel phase is markedly higher than that on the liquid phase.

It is useful to compare the results presented above with the results obtained in a work performed by Picas et al.(2008) on a similar system: a supported POPE bilayer. They attributed the variations in the jump-through force value with temperature also to a transition from the liquid crystalline phase to the inverted hexagonal phase of the bilayer with the formation of intermediate stalk structures. It is to be stressed that in the work by Picas et al. the bilayer was assembled with the sequential Langmuir-Schaefer technique whereas, in the work by Alessandrini et al. (2011), the bilayer was assembled with the vesicle fusion technique. It is possible that the two preparations methods leads to different behaviors of the supported lipid bilayers.

All the discussion on the temperature dependence is relevant for functional activity of membrane proteins. In fact, many membrane proteins partition preferentially in one type of domain when a phase coexistence state is present in the lipid bilayer (Mall et al., 2001; Baldwin and Hubbell, 1985). The preferential partitioning determines the environment around the membrane protein, including the mechanical properties of the bilayer and the hydrophobic matching condition. In the specific case of a $\mathrm{K}^{+}$ion channel, KcsA, it has been demonstrated by AFM imaging that, upon phase transition of the hosting lipid bilayer between the liquidcrystalline to the gel phase, the proteins preferentially localize in the liquid-crystalline domains (Seeger et al., 2009b). In these domains, while the bilayer is in the coexistence region of the transition, the proteins will experience an increased lateral area expansion coefficient of the bilayer. By performing a functional test of KcsA in a planar lipid bilayer (voltage clamp measurements on Black Lipid Membranes) it has been demonstrated that, when the lipid bilayer is in the phase transition region, all the functional parameters of the KcsA channel, at the single molecule level, change (Seeger et al., 2010b). In particular, the functional parameters follow the trend of the excess heat capacity of the lipid bilayer as a function of the temperature. Given that the heat capacity of a system is strictly related to the fluctuations of its parameters, the scenario of the mechanical properties of the lipid bilayers influencing the behavior of membrane proteins appears consistent Obviously, in the case of a multicomponent lipid bilayer, phase transition will produce a redistribution of the relative lipid composition in each different domain. This phenomenon will point also to the role of the specific lipid composition on the membrane protein activity. However, even if this role is definitely relevant, in the specific case at issue, it has been demonstrated that the effect of the enrichment of the domains hosting the proteins in the lipid favoring an increased activity of the channel cannot explain the magnitude of the observed enhanced activity (Seeger et al., 2010b). The overall picture for the KcsA in the POPE:POPG lipid mixture appears as follows: (1) upon the beginning of a phase transition, KcsA proteins preferentially distribute in the still liquid-crystalline domains; (2) these domains undergo a strong increase of fluctuations and of their compressibility at the nanometer scale as demonstrated by Atomic Force Spectroscopy; (3) proteins undergo variations of their activity when the lipid bilayer is in the phase transition region and the strength of these variations scales with the excess heat capacity profile of the bilayer as a function of temperature. The observed behavior, even if it cannot be extrapolated to the entire plasma membrane, shows the relevance of the physical state of the bilayer in affecting the transmembrane protein behavior.

The interleaflet coupling in lipid bilayers is at present a phenomenon investigated from both an experimental and theoretical point of view. The relevance of this phenomenon stems from its possible role as a lipid signaling mechanism between the two leaflets of a bilayer and, consequently, between the inner and outer regions of a cell. In the description of the chemical-physical properties of SLBs it has been stated that the presence of the support can induce vertical asymmetries in the two leaflets affecting their coupling. According to different preparation procedures and to ionic strength of the solution the degree of interleaflet coupling can be modulated (Seeger et al., 2009a). Does the degree of interleaflet coupling have an effect on the features of a force curve on supported lipid bilayers? It has been shown that in some cases the force curves on lipid bilayers present two jump-through events. Even if one cannot exclude that the two jump-through events are 


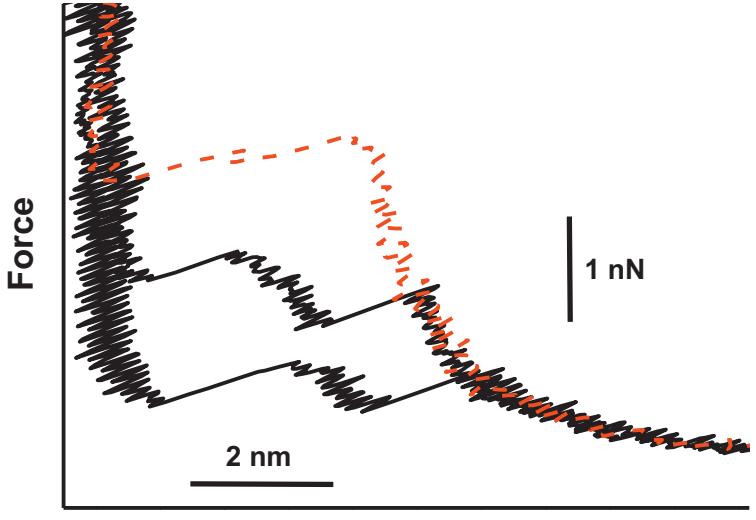

Distance

Fig. 6. Force spectroscopy curves obtained on a POPE supported lipid bilayer assembled by a procedure which results in the bilayer with uncoupled leaflets. The two black curves (continuous lines) have been obtained with a tip speed of $624 \mathrm{~nm} / \mathrm{s}$ whereas the red curve (dotted line) has been obtained with a tip speed of $1200 \mathrm{~nm} / \mathrm{s}$. The presence of two jump-through events can be associated with the sequential penetration of the AFM tip through the two lipid leaflets. (For interpretation of the references to color in this figure legend, the reader is referred to the web version of the article.)

associated with the presence of two bilayers, one on the substrate and the other one on the tip surface (Franz et al., 2002; Pera et al., 2004), it must be also considered that the two events could be due to the sequential penetration of the tip through the two leaflets. It can be shown that the possibility of observing two events in the same curve is strongly related to the degree of interleaflet coupling in the bilayer: strongly coupled leaflets lead to only one jump-through event, whereas decoupled leaflets, which show two independent phase transitions, can give rise to two events. In the latter case, considering the involved vertical distance for each jump, the presence of two events can be most likely attributed to the sequential penetration of the two leaflets. Fig. 6 shows an example of force curves on a POPE bilayer assembled on mica so to have the two leaflets uncoupled. The presence of two events, each one associated with one leaflet, is evident. Moreover, considering that the second sequential event should be the jump through the proximal leaflet, the higher value of jump-through force is consistent with its higher lipid surface density (Grant and Tiberg, 2002). It can also be demonstrated that, in case of intermediate coupling strength, the possibility of observing only one event or two events is connected to the speed of the tip during the force-indentation run. This possibility points to the role of the molecular details of the jump-through event.

As already pointed out, variations of parameters other than temperature can affect the mechanical stability of lipid bilayers. Among these, the ionic strength of the solution has been largely investigated (Garcia-Manyes et al., 2005b; Alessandrini et al., 2011). In this context both divalent cations and monovalent ions have been considered. It is well established that divalent cations can have, also at very low concentration, strong effects on the organization of bilayers (Pabst et al., 2007; Rand and Sengupta, 1972). On the contrary, it is usually assumed that monovalent ions can significantly affect the structure of lipid bilayers only at very high concentration (Pabst et al., 2007). A phenomenon which is usually observed in experiments performed on supported lipid bilayer is that the jump-through force increases with the ionic strength of the solution. This behavior is typically justified supposing that an increase of the ionic strength induces a lateral compaction of the lipids and an increase of the required punch-through force (Gurtovenko and Vattulainen, 2008). Molecular Dynamic simulations of the effects of monovalent ions on phospholipid bilayers revealed that the effect of the ions on the structure of the bilayer depends on the tendency of specific headgroups to form both intraand extra-molecular hydrogen bonds and on the specific ion at issue (Gurtovenko and Vattulainen, 2008). However, it should be noted that, in some cases, results from MD simulations and corresponding experimental data lack in consistency (Ferber et al., 2011). It is evident that the effect of ions should strongly depend on the overall charge in lipid headgroups, being strongest for more charged lipids. The effect of monovalent ions on supported lipid bilayers has been studied in the case of both charged and zwitterionic phospholipids (Alessandrini et al., 2011). A useful model system to compare the results with is represented by lipid monolayers in Langmuir trough. Fig. 7 shows the effect of an increasing concentration of $\mathrm{KCl}$ on the punch-through force for a POPG supported bilayer. The PG headgroup is negatively charged for $\mathrm{pH}$ values around neutrality. It is evident that the value of the jump-through force increases with ion concentration without reaching a saturation value up to $50 \mathrm{mM} \mathrm{KCl}$. A similar behavior is observed for a zwitterionic headgroup such as PE (Alessandrini et al., 2011). Measuring the lateral compressibility on lipid monolayers it appears that, whereas in the case of POPG the variation of the $\mathrm{KCl}$ concentration in the subphase produces evident effects, in the case of POPE these effects are negligible (Alessandrini et al., 2011). In the case of lipid monolayers the obtained results are easily interpreted on the basis of the effect of ions on the lateral interaction between the lipid headgroups, but the same interpretation scheme is incomplete for understanding the stability of supported lipid bilayers as probed by the jumpthrough force value. A more complete explanation of the observed behavior for supported lipid bilayer should include also the effect of the ions in solution on the presence of a transmembrane potential drop across the lipid bilayer. In fact, supported bilayers are usually assembled on charged surfaces. The electrostatic potential in solution produced by the charged substrate is characterized by a decay distance (Debye length) depending on the ionic strength of the solution. Accordingly, an increase of the ion concentration in solution decreases the decay distance and could decrease the value of the potential drop across the bilayer (Shreve et al., 2008). Given the electrostrictive properties of lipid bilayers (Petrov and Usherwood, 1994), a decrease of the voltage drop will increase the force required to jump-through the bilayer.

As we already pointed out, the force spectroscopy technique appears as a valuable method to distinguish different regions of the lipid bilayer according to their different mechanical properties (Sullan et al., 2009). This is true both in the case of gel and liquidcrystalline domains and in the case of liquid disordered and liquid ordered domains. The last case is usually associated with ternary lipid mixtures involving cholesterol. A recent study by Sullan et al. (2010) concentrated on the mechanical stability of coexistent liquid domains in supported lipid bilayers composed by DOPC/SM/Chol. They measured the mechanical properties of both liquid disordered and liquid ordered domains as a function of cholesterol concentration establishing that an increase of cholesterol concentration reduces the mechanical stability of the domains consistently with the fluidizing effect of cholesterol.

\section{Dynamic force spectroscopy of supported lipid bilayers}

As we pointed out in Section 1, the phenomenon of jumpthrough in SLBs is a dynamic process. As such, the force value depends on the force application rate. Considering the process of jump-through as a chemical reaction, it proceeds with a random walk on the potential energy curve describing the route to the intermediate state. In the case of a higher force application rate, the system will have less time to explore the conformation landscape and a higher force will be reached before the tip will be able 

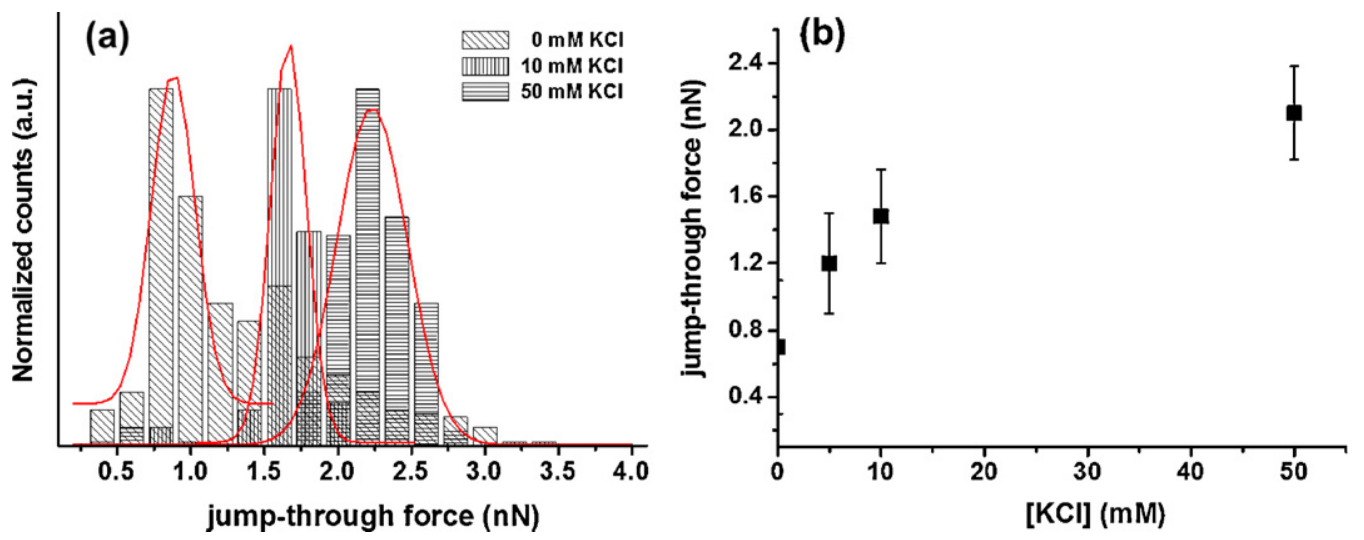

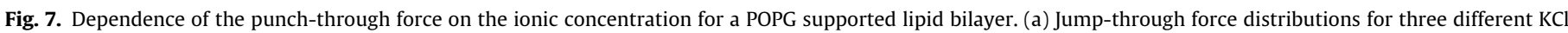
concentrations (the lines are Gaussian fits to the data); (b) overall trend of the most probable jump-through force from 0 to $50 \mathrm{mM} \mathrm{KCl}$ concentration.

to penetrate the bilayer (Bell, 1978; Evans and Ritchie, 1997). This is a phenomenological description, which captures the qualitative feature of the process. Furthermore, dynamic force spectroscopy allows exploring the energy landscape describing the penetration process, at least from the initial state to the intermediate one (Hummer and Szabo, 2003). In this case, the dynamic experiments provide an exciting possibility of studying the jump-through event and extracting parameters for the same process in the absence of force (Dudko et al., 2006). Fig. 8 shows the results of a dynamic force spectroscopy experiment on a supported POPE bilayer in liquid-crystalline phase. The plot reports the most probable jumpthrough force value as a function of the tip speed in semilogarithmic scale. The linear relation between the two parameters is consistent with the phenomenological description we mentioned above. One of the problems of a theoretical description of dynamic force spectroscopy experiments is related to the limited tip speed range experimentally investigated. A microscopic description of dynamic force spectroscopy experiments could foresee a more complex relation between the most probable force for the process at issue and the tip speed in a larger range of tip speeds, mainly by removing the restriction of a fixed position for the location of the transition state (Hummer and Szabo, 2003; Dudko et al., 2003, 2006).

Different theoretical models have been developed to interpret dynamic force spectroscopy experiments and, moreover, some specific models have also been developed in the context of punchthrough experiments on supported lipid bilayers (Butt and Franz, 2002; Loi et al., 2002; Franz et al., 2002). One of the main goals

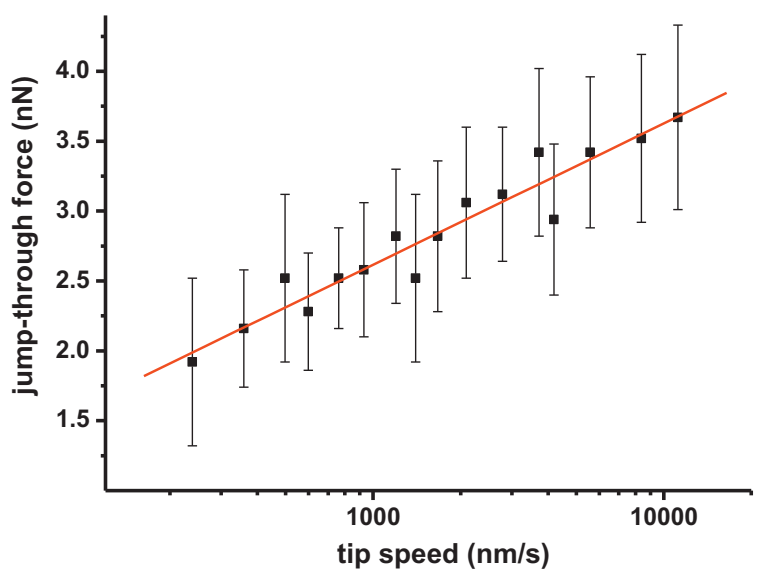

Fig. 8. Punch-through force values on a POPE bilayer in $50 \mathrm{mM} \mathrm{KCl}$ at $25^{\circ} \mathrm{C}$ as a function of the tip speed. The error bars represent the standard deviations of the measurements. of theories describing the effect of a force on the occurrence of a process is that of extrapolating the parameters that describe the process in the absence of an applied force. Among these parameters, the most important ones are the activation energy for the process $\left(\Delta G^{0}\right)$ and the rate of the process at zero force $\left(k_{\text {off }}^{0}\right)$. One of these is the Bell-Evans model, usually referred to as the phenomenological model (see above) (Evans and Ritchie, 1997). This model foresees a linear relation between the most probable collapsing force and the logarithm of the loading rate. A fit of this model to the experimental data allows retrieving $k_{\text {off }}^{0}$ and the distance to the transition state $\Delta x . k_{o f f}^{0}$ represents the rate of the process which, in the case at issue, involves the formation of a hole in the bilayer large enough for the tip to penetrate at zero force, whereas $\Delta x$ should represent the distance between the initial equilibrium state and the intermediate reaction state, characterized by the highest energy value along the transition to the final state. Abdulreda and Moy (2007) exploited the phenomenological theory to analyze an event involving the fusion of two lipid bilayers studied by dynamic force spectroscopy. In this work they obtained a linear relation between the force required for the fusion process and the logarithm of the loading rate. From their data a value for the distance to the transition state in the order of $10^{-2} \mathrm{~nm}$ was obtained by exploiting the phenomenological Bell-Evans model. It is to be stressed that the values which can be extracted from the fits are obtained from theories which have been developed in the context of a single bond and which assumes the reaction coordinate as an unidimensional parameter in the direction defined by the pulling or pushing path of the tip (Best et al., 2008). Complicated events such as the rupture of a lipid bilayer and the penetration of the AFM tip could require a description in terms of multiple reaction coordinates. This is particularly true in the case of supported lipid bilayers in which the force is applied in the direction perpendicular to the plane of the bilayer and the interactions involved in the collapse of the structure act mainly in the direction parallel to the plane of the membrane. In particular, the value for the distance to the transition state obtained by Abdulreda and Moy is too small for assuming a straightforward physical meaning. Its exceedingly small value means that the overall process is almost insensitive to the application of a force, but such a value seems also unable to separate significantly the two equilibrium states of the transition. That figure is probably affected by the fact that the assumed reaction coordinate (the pushing direction of the tip) does not correspond to the best describing reaction coordinate for the process (Best et al., 2008).

The molecular model proposed by Butt (Butt and Franz, 2002) allows extracting both the activation energy for the process and $k_{\text {off }}^{0}$, but it does not allow the extraction of the distance to the transition state. In the paper by Sullan et al. (2010), this model has been 
exploited to extract the activation energies for the formation of pores by the AFM tip in supported lipid bilayers including cholesterol. They obtained results which were in a remarkable accord with the data obtained for the fusion process of biomembranes and model membranes.

Another model developed by Butt and Franz is the continuous nucleation one. Within this model the distribution of the force values for the jump-through phenomenon can be exploited to obtain information on parameters such as the line tension of the bilayer and the "spreading pressure", which represents the tendency of the film to spread into the gap between the tip and substrate (GarciaManyes et al., 2010).

Models that account for a microscopic description of the system are mainly derived by the transition state theory and the Kramer's approximation assuming a specific analytical description of the energy landscape for the process. These models have the advantage of providing directly an estimate of the activation energy for the process. Future works will establish if the process of AFM tip jump-through in supported lipid bilayers can really be described by these theories and they will also establish the physical meaning of the parameters which will eventually be available from fitting the theories to the experimental data.

\section{Conclusions and future perspectives}

We showed how force spectroscopy experiments on supported lipid bilayers provide useful information about the mechanical properties of the bilayer at the nanoscale. This is true both for mechanical stability of the bilayer and mechanical moduli (spring constants) of the membrane. Obviously, in the second case care should be paid to the fact that the influence of the rigid substrate immediately below the very thin $(\sim 5 \mathrm{~nm})$ lipid bilayer can alter the quantitative evaluation of the mechanical moduli (Dimitriadis et al., 2002). The development of contact mechanics theory specifically designed to consider the molecular structure of lipid bilayers and the interactions of both the hydrophobic and polar regions of the lipids could increase the accuracy of the extracted parameters. For example, the use of cantilevers with tips shaped as microspheres could allow the application of measurable forces with a small deformation of the sample. In this case the sample deformation could remain in the linear elastic regime and the extraction of a parameter representing the spring constant for the deformation of the bilayer would be more reliable. The understanding of the molecular deformation induced on a lipid bilayer by the AFM tip would surely benefit from molecular dynamic simulation of the process. This could also describe the relation between the compression modulus of a lipid bilayer and its lateral expansion modulus, which are probably both involved in the indentation measurements performed by AFM. Further theoretical and experimental efforts will also help clarifying the role of the viscosity properties of the bilayer. In the case of experimental efforts it is to be highlighted the introduction of a new technique based on indentation force spectroscopy which allows the acquisition of maps of the mechanical properties of a sample at high resolution (Rico et al., 2012). The same techniques has been also recently applied to the determination of the mechanical properties of a supported lipid bilayer in terms of elastic moduli (Picas et al., 2012). All these studies will also allow an easier comparison between the nanoscale properties and the mesoscale ones measured by the micropipette aspiration technique.

It should be however considered that many of the considerations developed in this review refer to model systems of the biological membrane. Whereas in model systems the presence of "lipid rafts" interpreted in the sense of transient compartmentalization of lipids and proteins in micro/nanometric domains is well established, in cell membranes, the observation of "lipid rafts" has remained elusive so far. Indirect evidence of their presence has been obtained by biophotonics techniques but imaging evidences still lack (Eggeling et al., 2009). The investigation on model systems is however very useful also for the validation of experimental approaches which can be extended to establish the presence of nanodomains in biological membranes.

Another important field of study is connected to how molecules, which can interact with the lipid bilayer, are able to affect the mechanical properties of lipid bilayers as measured by AFM. In the literature it is possible to find some examples in which proteins, such as cytochrome c, which adsorb on the surface of lipid bilayers, have been demonstrated to affect the mechanical stability of lipid bilayers on the nanoscale (Morandat and El Kirat, 2011). Another interesting case is that of the presence of amphipilic molecules or peptides, which can insert in the lipid bilayer and modify its mechanical parameters. For example, it has been shown by atomic force spectroscopy that a Bax-derived peptide is able to modify the line tension of a lipid bilayer (García-Sáez et al., 2007). This result can shed light on the mechanism of pore formation in lipid bilayers by many peptides. In general, atomic force spectroscopy could be exploited to study how amphiphilic drugs are able to affect the mechanical properties of a lipid bilayer and, as a consequence, the activity of membrane proteins. One of the techniques which is currently being used to monitor the mechanical changes induced by a drug on a lipid bilayer is based on the alterations that the studied drug produces on the activity of a wellknown ion channel: gramicidin (Lundbaek, 2008). The exploitation of force spectroscopy technique on lipid bilayers could complement the studies on gramicidin and provide a direct measurement of the spring constant associated with membrane deformations.

In the context of the energy landscape which describes the membrane rupture event, further theoretical developments, including a multidimensional description of the process in terms of reaction coordinates and further studies aimed at enhancing the explored force rate increase range, will help understanding some aspects that are still controversial.

The reviewed technique of force spectroscopy on supported lipid bilayers has already provided useful information on this biological membrane model system and promises to offer other valuable data in the near future. As it is typical of the biophysical approach, the choice of models of increasing complexity is to be followed and the final goal will be that of implementing this technique directly on biological membranes.

\section{Acknowledgement}

Authors acknowledge partial financial support by the Italian MIUR FIRB Project "Italnanonet".

\section{References}

Abdulreda, M.H., Moy, V.T., 2007. Atomic force microscope studies of the fusion of floating lipid bilayers. Biophys. J. 92, 4369-4378.

Alessandrini, A., Facci, P., 2005. AFM: a versatile tool in biophysics. Meas. Sci. Technol. 16, R65-R92.

Alessandrini, A., Facci, P., 2011a. Unraveling lipid/protein interaction in model lipid bilayers by atomic force microscopy. J. Mol. Recognit. 24, 387-396.

Alessandrini, A., Facci, P., 2011b. Changes in single $\mathrm{K}^{+}$channel behavior induced by a lipid phase transition. Commun. Integr. Biol. 4, 346-348.

Alessandrini, A., Seeger, H.M., Di Cerbo, A., Caramaschi, T., Facci, P., 2011. What do we really measure in AFM punch-through experiments on supported lipid bilayers? Soft Matter 7, 7054-7064.

Alessandrini, A., Seeger, H.M., Caramaschi, T., Facci, P., submitted for publication.

Armond, J.W., Macpherson, J.V., Turner, M.S., 2011. Pulling nanotubes from supported bilayers. Langmuir 27, 8269-8274.

Bagatolli, L.A., Ipsen, J.H., Simonsen, A.C., Mouritsen, O.G., 2010. An outlook on organization of lipids in membranes: searching for a realistic connection with the organization of biological membranes. Prog. Lipid Res. 49, 378-389. 
Baldwin, P.A., Hubbell, W.L., 1985. Effects of lipid environment on the light-induced conformational changes of rhodopsin. 2. Roles of lipid chain length, unsaturation, and phase state. Biochemistry 24, 2633-2639.

Beece, D., Eisenstein, L., Frauenfelder, H., Good, D., Marden, M.C., Reinisch, L., Reynolds, A.H., Sorensen, L.B., Yue, K.T., 1980. Solvent viscosity and protein dynamics. Biochemistry 19, 5147-5157.

Bell, G.I., 1978. Models for the specific adhesion of cells to cells. Science 200, 618-627.

Best, R.B., Paci, E., Hummer, G., Dudko, O.K., 2008. Pulling direction as a reaction coordinate for the mechanical unfolding of single molecules. J. Phys. Chem. B 112, 5968-5976.

Bier, M., Gowrishankar, T.R., Chen, W., Lee, R.C., 2004. Electroporation of a lipid bilayer as a chemical reaction. Bioelectromagnetics 25, 634-637.

Brian, A.A., McConnell, H.M., 1984. Allogeneic stimulation of cytotoxic T cells by supported planar membranes. Proc. Natl. Acad. Sci. U.S.A. 81, 6159-6163.

Butt, H.J., 1991a. Electrostatic interaction in atomic force microscopy. Biophys. J. 60, 777-785.

Butt, H.J., 1991b. Measuring electrostatic, van der Waals, and hydration forces in electrolyte solutions with an atomic force microscope. Biophys. J. 60, 1438-1444.

Butt, H.J., 1992. Measuring local surface charge densities in electrolyte solutions with a scanning force microscope. Biophys. J. 63, 578-582.

Butt, H.J., Franz, V., 2002. Rupture of molecular thin films observed in atomic force microscopy. I. Theory. Phys. Rev. E 66, 031601.

Castellana, E.T., Cremer, P.S., 2006. Solid supported lipid bilayers: from biophysical studies to sensor design. Surf. Sci. Rep. 61, 429-444.

Cevc, G., 1990. Membrane electrostatics. Biochim. Biophys. Acta 1031, 311-382.

Cevc, G., 1991. Isothermal lipid phase transitions. Chem. Phys. Lipids 57, 293-307.

Charrier, A., Thibaudau, F., 2005. Main phase transitions in supported lipid singlebilayer. Biophys. J. 89, 1094-1101.

Collins, M.D., 2008. Interleaflet coupling mechanisms in bilayers of lipids and cholesterol. Biophys. J. 94, L32-L34.

Collins, M.D., Keller, S.L., 2008. Tuning lipid mixtures to induce or suppress domain formation across leaflets of unsupported asymmetric bilayers. Proc. Natl. Acad. Sci. U.S.A. 105, 124-128.

Crane, J.M., Kiessling, V., Tamm, L.K., 2005. Measuring lipid asymmetry in planar supported bilayers by fluorescence interference contrast microscopy. Langmuir 21, 1377-1388.

Das, C., Sheikh, K.H., Olmsted, P.D., Connell, S.D., 2010. Nanoscale mechanical probing of supported lipid bilayers with atomic force microscopy. Phys. Rev. E 82, 041920.

Deserno, M., 2009. Mesoscopic membrane physics: concepts, simulations, and selected applications. Macromol. Rapid Commun. 30, 752-771.

Dieluweit, S., Csiszár, A., Rubner, W., Fleischhauer, J., Houben, S., Merkel, R., 2010. Mechanical properties of bare and protein-coated giant unilamellar phospholipid vesicles. A comparative study of micropipet aspiration and atomic force microscopy. Langmuir 26, 11041-11049.

Dimitriadis, E.K., Horkay, F., Maresca, J., Kachar, B., Chadwick, R.S., 2002. Determination of elastic moduli of thin layers of soft material using the atomic force microscope. Biophys. J. 82, 2798-2810.

Domke, J., Radmacher, M., 1998. Measuring the elastic properties of thin polymer films with the atomic force microscope. Langmuir 14, 3320-3325.

Dudko, O.K., Filippov, A.E., Klafter, J., Urbakh, M., 2003. Beyond the conventional description of dynamic force spectroscopy of adhesion bonds. Proc. Natl. Acad. Sci. U.S.A. 100, 11378-11381.

Dudko, O.K., Hummer, G., Szabo, A., 2006. Intrinsic rates and activation free energies from single-molecule pulling experiments. Phys. Rev. Lett. 96, 108101.

Dufrêne, Y.F., Barger, W.R., Green, J.B.D., Lee, G.U., 1997. Nanometer-scale surface properties of mixed phospholipid monolayers and bilayers. Langmuir 13, 4779-4784.

Dufrêne, Y.F., Boland, T., Schneider, J.W., Barger, W.R., Lee, G.U., 1998. Characterization of the physical properties of model biomembranes at the anometer scale with the atomic force microscope. Faraday Discuss. 111, 79-94.

Eggeling, C., Ringemann, C., Medda, R., Schwarzmann, G., Sandhoff, K., Polyakova, S., Belov, V.N., Hein, B., von Middendorff, C., Schönle, A., Hell, S.W., 2009. Direct observation of the nanoscale dynamics of membrane lipids in a living cell. Nature 457, 1159-1162.

Evans, E., Ritchie, K., 1997. Dynamic strength of molecular adhesion bonds. Biophys. J. 72, 1541-1555.

Feng, Z.V., Spurlin, T.A., Gewirth, A.A., 2005. Direct visualization of asymmetric behavior in supported lipid bilayers at the gel-fluid phase transition. Biophys. J. 88, 2154-2164.

Ferber, U., Kaggwa, G., Jarvis, S., 2011. Direct imaging of salt effects on lipid bilayer ordering at sub-molecular resolution. Eur. Biophys. J. 40, 329-338.

Franz, V., Loi, S., Muller, H., Bamberg, E., Butt, H.J., 2002. Tip penetration through lipid bilayers in atomic force microscopy. Colloids Surf. B Biointerfaces 23, 191-2002.

Frauenfelder, H., Chen, G., Berendzen, J., Fenimore, P.W., Jansson, H., McMahon, B.H., Stroe, I.R., Swenson, J., Young, R.D., 2009. A unified model of protein dynamics. Proc. Natl. Acad. Sci. U.S.A. 106, 5129-5134.

Frauenfelder, H., 2010. In: Chan, S.S., Chan, W.S. (Eds.), The Physics of Proteins: An Introduction to Biological Physics and Molecular Biophysics.

Garcia-Manyes, S., Oncins, G., Sanz, F., 2005a. Effect of temperature on the nanomechanics of lipid bilayers studied by force spectroscopy. Biophys. J. 89, 4261-4274.

Garcia-Manyes, S., Oncins, G., Sanz, F., 2005b. Effect of ion-binding and chemical phospholipid structure on the nanomechanics of lipid bilayers studied by force spectroscopy. Biophys. J. 89, 1812-1826.

Garcia-Manyes, S., Gorostiza, P., Sanz, F., 2006. Titration force microscopy on supported lipid bilayers. Anal. Chem. 78, 61-70.
Garcia-Manyes, S., Sanz, F., 2010. Nanomechanics of lipid bilayers by force spectroscopy with AFM: a perspective. Biochim. Biophys. Acta 1798, 741-749.

Garcia-Manyes, S., Redondo-Morata, L., Oncins, G., Sanz, F., 2010. Nanomechanics of lipid bilayers: heads or tails? J. Am. Chem. Soc. 132, 12874-12886.

García-Sáez, A.J., Chiantia, S., Salgado, J., Schwille, P., 2007. Pore formation by a Baxderived peptide: effect on the line tension of the membrane probed by AFM Biophys. J. 93, 103-112.

Glaser, R.W., Leikin, S.L., Chernomordik, L.V., Pastushenko, V.F., Sokirko, A.I., 1988 Reversible electrical breakdown of lipid bilayers: formation and evolution of pores. Biochim. Biophys. Acta 940, 275-287.

Grant, L.M., Tiberg, F., 2002. Normal and lateral forces between lipid covered solids in solution: correlation with layer packing and structure. Biophys. J. 82, 1373-1385.

Gurtovenko, A.A., Vattulainen, I., 2008. Effect of $\mathrm{NaCl}$ and $\mathrm{KCl}$ on phosphatidylcholine and phosphatidylethanolamine lipid membranes: insight from atomic-scale simulations for understanding salt-induced effects in the plasma membrane. J. Phys. Chem. B 112, 1953-1962.

Heimburg, T., 1998. Mechanical aspects of membrane thermodynamics. Estimation of the mechanical properties of lipid membranes close to the chain melting transition from calorimetry. Biochim. Biophys. Acta 1415, 147-162.

Heimburg, T., 2010. Lipid ion channels. Biophys. Chem. 150, 2-22.

Helfrich, W., 1973. Elastic properties of lipid bilayers-theory and possible experiments. Z. Naturforsch. C 28, 693-703.

Henriksen, R., Ipsen, J.H., 2004. Measurement of membrane elasticity by micropipette aspiration. Eur. Phys. J. E 14, 149-167.

Hertz, H., 1882. Über die Berührung fester, elastischer Körper. J. Reine Angew Math., $156-171$.

Hetzer, M., Heinz, S., Grage, S., Bayerl, T.M., 1998. Asymmetric molecular friction in supported phospholipid bilayers revealed by NMR measurements of lipid diffusion. Langmuir 14, 982-984.

Hoffman, B.D., Crocker, J.C., 2009. Cell mechanics: dissecting the physical responses of cells to force. Annu. Rev. Biomed. Eng. 11, 259-288.

Hummer, G., Szabo, A., 2003. Kinetics from nonequilibrium single-molecule pulling experiments. Biophys. J. 85, 5-15.

Israelachvili, J., 1992. Intermolecular and Surface Forces. Academic Press, New York.

Johnson, S.J., Bayerl, T.M., McDermott, D.C., Adam, G.W., Rennie, A.R., Thomas, R.K., Sackmann, E., 1991. Structure of an adsorbed dimyristoylphosphatidylcholine bilayer measured with specular reflection of neutrons. Biophys. J. 59, 289-294.

Kalani, J.S., Pandeya, A.P., Haquea, F., Proctora, E.A., Ribbea, A.E., Hovis, J.S., 2007 Effect of surface treatment on diffusion and domain formation in supported lipid bilayers. Biophys. J. 92, 2445-2450.

Kasas, S., Dietler, G., 2008. Probing nanomechanical properties from biomolecules to living cells. Pflugers Arch. Eur. J. Physiol. 456, 13-27.

Keller, D., Larsen, N.B., Moller, I.M., Mouritsen, O.G., 2005. Decoupled phase transitions and grain-boundary melting in supported phospholipid bilayers. Phys. Rev. Lett. 94, 25701.

Kim, J., Kim, G., Cremer, P.S., 2001. Investigations of water structure at the solid/liquid interface in the presence of supported lipid bilayers by vibrational sum frequency spectroscopy. Langmuir 17, 7255-7260.

Koenig, B.W., Kruger, S., Orts, W.J., Majkrzak, C.F., Berk, N.F., Silverton, J.V., Gawrisch, K., 1996. Neutron reflectivity and atomic force microscopy studies of a lipid bilayer in water adsorbed to the surface of a silicon single crystal. Langmuir 12, 1343-1350.

Kunneke, S., Kruger, D., Janshoff, A., 2004. Scrutiny of the failure of lipid membranes as a function of headgroups, chain length, and lamellarity measured by scanning force microscopy. Biophys. J. 86, 1545-1553.

Lee, A.G., 2004. How lipids affect the activities of integral membrane proteins. Biochim. Biophys. Acta 1666, 62-87.

Lin, W.-C., Blanchette, C.D., Ratto, T.V., Longo, M.L., 2006. Lipid asymmetry in DLPC/DSPC-supported lipid bilayers: a combined AFM and fluorescence microscopy study. Biophys. J. 90, 228-237.

Lingwood, D., Simons, K., 2010. Lipid rafts as a membrane-organizing principle. Science 327, 46-50.

Loi, S., Sun, G., Franz, V., Butt, H.J., 2002. Rupture of molecular thin films observed in atomic force microscopy. II. Experiment. Phys. Rev. E 66, 031602.

Lundbaek, J.A., 2008. Lipid bilayer-mediated regulation of ion channel function by amphiphilic drugs. J. Gen. Physiol. 131, 421-429.

Mall, S., Broadbridge, R., Sharma, R.P., East, J.M., Lee, A.G., 2001. Self-association of model transmembrane alpha-helices is modulated by lipid structure. Biochemistry $40,12379-12386$.

Marsh, D., 2008. Protein modulation of lipids, and vice-versa, in membranes. Biochim. Biophys. Acta 1778, 1545-1575.

Martinac, B., Buechner, M., Delcour, A.H., Adler, J., Kung, C., 1987. Pressure-sensitive ion channel in Escherichia coli. Proc. Natl. Acad. Sci. U.S.A. 84, 2297-2301.

McConnell, H.M., Watts, T.H., Weis, R.M., Brian, A.A., 1986. Supported planar membranes in studies of cell-cell recognition in the immune system. Biochim. Biophys. Acta 864, 95-106.

McIntosh, T.J., Simon, S.A., 2006. Roles of bilayer material properties in function and distribution of membrane proteins. Annu. Rev. Biophys. Biomol. Struct. 35, 177-198.

Merkel, R., Sackmann, E., Evans, E., 1989. Molecular friction and epitactic coupling between monolayers in supported bilayers. J. Phys. France 50, 1535-1555.

Mey, I., Stephan, M., Schmitt, E.K., Müller, M.M., Ben Amar, M., Steinem, C., Janshoff, A., 2009. Local membrane mechanics of pore-spanning bilayers. J. Am. Chem. Soc. 131, 7031-7039.

Mora, T., Bialek, W., 2011. Are biological systems poised at criticality? J. Stat. Phys. $144,268-302$. 
Morandat, S., El Kirat, K., 2011. Cytochrome c provokes the weakening of zwitterionic membranes as measured by force spectroscopy. Colloids Surf. B: Biointerfaces $82,111-117$.

Morrisett, J.D., Pownall, H.J., Plumlee, R.T., Smith, L.C., Zehner, Z.E., 1975. Multiple thermotropic phase transitions in Escherichia coli membranes and membrane lipids. A comparison of results obtained by nitroxyl stearate paramagnetic resonance, pyrene excimer fluorescence, and enzyme activity measurements. J. Biol. Chem. 250, 6969-6976.

Neuman, K.C., Nagy, A., 2008. Single-molecule force spectroscopy: optical tweezers, magnetic tweezers and atomic force microscopy. Nat. Methods 5, 491-505.

Pabst, G., Hodzic, A., Strancar, J., Danner, S., Rappolt, M., Laggner, P., 2007. Rigidification of neutral lipid bilayers in the presence of salts. Biophys. J. 93, 2688-2696.

Pelling, A.E., Horton, M.A., 2008. An historical perspective on cell mechanics. Pflugers Arch. 456, 3-12.

Pera, I., Stark, R., Kappl, M., Butt, H.J., Benfenati, F., 2004. Using the atomic force microscope to study the interaction between two solid supported lipid bilayers and the influence of synapsin I. Biophys. J. 87, 2446-2455

Petrov, A.G., Usherwood, P.N.R., 1994. Mechanosensitivity of cell membranes. Eur. Biophys. J. 23, 1-19.

Phillips, R., Ursell, T., Wiggins, P., Sens, P., 2009. Emerging roles for lipids in shaping membrane-protein function. Nature 459, 379-385.

Picas, L., Montero, M.T., Morros, A., Oncins, G., Hernández-Borrell, J., 2008. Phase Changes in Supported Planar Bilayers of 1-Palmitoyl-2-oleoyl-sn-glycero-3phosphoethanolamine. J. Phys. Chem. B 112, 10181-10187.

Picas, L., Suárez-Germà, C., Teresa Montero, M., Hernández-Borrell, J., 2010. Force spectroscopy study of Langmuir-Blodgett asymmetric bilayers of phosphatidylethanolamine and phosphatidylglycerol. J. Phys. Chem. B 114, 3543-3549.

Picas, L., Rico, F., Scheuring, S., 2012. Direct measurement of the mechanical properties of lipid phases in supported bilayers. Biophys. J. 102, L01-L3.

Przybylo, M., Sýkora, J., Humpolíĉková, J., Benda, A., Zan, A., Hof, M., 2006. The lipid diffusion in giant unilamellar vesicles is more than two times faster than in supported phospholipid bilayers under identical conditions. Langmuir 22 9096-9099.

Radmacher, M., Fritz, M., Kacher, C.M., Cleveland, J.P., Hansma, P.K., 1996. Measuring the viscoelastic properties of human platelets with the atomic force microscope. Biophys. J. 70, 556-567.

Rand, R.P., Sengupta, S., 1972. Cardiolipin forms hexagonal structures with divalent cations. Biochim. Biophys. Acta 255, 484-492.

Rawicz, W., Olbrich, K.C., McIntosh, T., Needham, D., Evans, E., 2000. Effect of chain length and unsaturation on elasticity of lipid bilayers. Biophys. J. 79, 328-339.

Rico, F., Su, C., Scheuring, S., 2012. Mechanical mapping of single membrane proteins at submolecular resolution. Nano Lett. 11,3983-3986.
Sachs, F., 2010. Stretch-activated ion channels: what are they? Physiology 25, 50-56 Seeger, H.M., Marino, G., Alessandrini, A., Facci, P., 2009a. Effect of physical parameters on the main phase transition of supported lipid bilayers. Biophys. J. 97 1067-1076.

Seeger, H.M., Bortolotti, C.A., Alessandrini, A., Facci, P., 2009b. Phase-transitioninduced protein redistribution in lipid bilayers. J. Phys. Chem. B 113 16654-16659.

Seeger, H.M., Di Cerbo, A., Alessandrini, A., Facci, P., 2010a. Supported lipid bilayers on mica and silicon oxide: comparison of the main phase transition behavior. J. Phys. Chem. B 114, 8926-8933.

Seeger, H.M., Aldrovandi, L., Alessandrini, A., Facci, P., 2010b. Changes in single $\mathrm{K}^{+}$ channel behavior induced by a lipid phase transition. Biophys. J. 99, 3675-3683.

Shreve, A.P., Howland, M.C., Sapuri-Butti, A.R., Allen, T.W., Parikh, A.N., 2008 Evidence for leaflet-dependent redistribution of charged molecules in fluid supported phospholipid bilayers. Langmuir 24, 13250-13253.

Simons, K., Ikonen, E., 1997. Functional rafts in cell membranes. Nature 387, 569-572.

Stottrup, B., Veatch, S., Keller, S., 2004. Nonequilibrium behavior in supported lipid membranes containing cholesterol. Biophys. J. 86, 2942-2950.

Sullan, R.M., Li, J.K., Zou, S., 2009. Direct correlation of structures and nanomechanical properties of multicomponent lipid bilayers. Langmuir 25, 7471-7477.

Sullan, R.M., Li, J.K., Hao, C., Walker, G.C., Zou, S., 2010. Cholesterol-dependent nanomechanical stability of phase-segregated multicomponent lipid bilayers. Biophys. J. 99, 507-516.

Thompson, D.W., 1917. On Growth and Form. Cambridge University Press, London.

Thormann, E., Simonsen, A.C., Nielsen, L.K., Mouritsen, O.G., 2007. Ligand-receptor interactions and membrane structure investigated by AFM and time-resolved fluorescence microscopy. J. Mol. Recognit. 20, 554-560.

Tokumasu, F., Jin, A.J., Dvorak, J.A., 2002. Lipid membrane phase behaviour elucidated in real time by controlled environment atomic force microscopy. J. Electron Microsc. 51, 1-9.

Voïtchovsky, K., Antoranz Contera, S., Kamihira, M., Watts, A., Ryan, J.F., 2006. Differential stiffness and lipid mobility in the leaflets of purple membranes. Biophys. J. 90, 2075-2085.

Xing, C., Faller, R., 2008. Interactions of lipid bilayers with supports: a coarse-grained molecular simulation study. J. Phys. Chem. B 112, 7086-7094.

Xing, C., Faller, R., 2009. Density imbalances and free energy of lipid transfer in supported lipid bilayers. J. Chem. Phys. 131, 175104

Yarrow, F., Vlugt, T.J.H., van der Eerden, J.P.J.M., Snel, M.M.E., 2005. Melting of a DPPC lipidbilayer observedwith atomic force microscopy andcomputer simulation. J. Cryst. Growth 275, e1417-e1421.

Zhang, L., Granick, S., 2005. Lipid diffusion compared in outer and inner leaflets of planar supported bilayers. J. Chem. Phys. 123, 211104-211107. 